\title{
Effect of Radiation, Organic Solvents and Vibration Occupational Exposure on the Thyroid Disease: Results from Project ELEFANT
}

\author{
Bibo Yuan ${ }^{1+}$, Citlalli Osorio-Yáñez ${ }^{2+}$, Junkai Fang ${ }^{3}$, Lei Zhao ${ }^{4,5,6}$, Ander Wilson? \\ Marco Sanchez-Guerra ${ }^{8}$, Hongbin Liu ${ }^{3}$, Peng-Hui Li ${ }^{9 *}$, Liqiong Guo ${ }^{4,5,6 *}$ \\ ${ }^{1}$ Department of Gynecology and Obstetrics, Tianjin Medical University General Hospital, Tianjin \\ 300052, China \\ ${ }^{2}$ Instituto de Investigaciones Biomédicas, Universidad Nacional Autónoma de México, México \\ DF 04510, Mexico \\ ${ }^{3}$ Tianjin Institute of Medical \& Pharmaceutical Sciences, Tianjin 300070, China \\ ${ }^{4}$ Institute of Disaster and Emergency Medicine, Tianjin University, Tianjin 300072, China \\ ${ }^{5}$ Tianjin Key Laboratory of Disaster Medicine Technology, Tianjin 300072, China \\ ${ }^{6}$ Wenzhou Safety (Emergency) Institute, Tianjin University, Wenzhou 325000, China \\ ${ }^{7}$ Department of Statistics, Colorado State University, Fort Collins, Colorado 80523, USA \\ ${ }^{8}$ Department of Developmental Neurobiology, National Institute of Perinatology, Mexico City \\ 04510, Mexico \\ ${ }^{9}$ School of Environmental Science and Safety Engineering, Tianjin University of Technology, \\ Tianjin 300384, China
}

\section{ABSTRACT}

\section{OPEN ACCESS}

Received: April 13, 2021

Revised: June 2, 2021

Accepted: June 4, 2021

\section{${ }^{*}$ Corresponding Authors:}

Peng-Hui Li

lipenghui406@163.com

Liqiong Guo

yingqidao@163.com

\section{${ }^{+}$These authors contributed} equally to this work

\section{Publisher:}

Taiwan Association for Aerosol Research

ISSN: $1680-8584$ print

ISSN: 2071-1409 online

(c) Copyright: The Author(s) This is an open access article distributed under the terms of the Creative Commons Attribution License (CC BY 4.0), which permits unrestricted use, distribution, and reproduction in any medium, provided the original author and source are cited.
Although occupational exposure to radiation and organic solvents has been proven to increase the chance of developing thyroid disease, one of the leading endocrine disorders, much less is known about the potential risk factor of vibration exposure. Thus, this study aimed to examine the relationships between occupational exposure to radiation, organic solvents and vibration, and thyroid disease risk and thyroid-stimulating hormone (TSH) levels by utilizing data sets from Project ELEFANT, which comprises 124,286 participants from Tianjin, China. We reviewed the medical records, including the thyroid disease history, of young adult participants and measured the TSH levels in the females via automated immunometric assay. Logistic models adjusted for confounders revealed significant associations between exposure to radiation (odds ratio [OR]: 1.73; 95\% confidence interval [Cl]: 1.1-2.7), organic solvents (OR: $2.0 ; 95 \% \mathrm{Cl}: 1.3-2.9$ ) and vibration (OR: $4.4 ; 95 \% \mathrm{Cl}: 2.0-8.75$ ), and an increased risk of thyroid disease. Additionally, the TSH concentrations in the female participants (median: $1.95 ; 95 \% \mathrm{Cl}: 2.45-2.77$ ) displayed a correlation exclusively with vibration exposure $(\beta: 2.80 ; 95 \% \mathrm{Cl}: 1.07-4.54)$. Our results show, for the first time, that vibration exposure is associated with an increased risk of thyroid disease and elevated TSH levels. If these findings are confirmed in other cohorts, thyroid examinations should be conducted for individuals exposed to vibration in the workplace.

Keywords: Vibration, Organic solvents, Radiation, Thyroid disease, Thyroid-stimulating hormone

\section{INTRODUCTION}

Thyroid disease is one of the leading endocrine disorders (Vanderpump, 2011). Thyroid diseases can be classified as structural or functional, with structural thyroid diseases including tumours (benign or malignant), nodules and goitre (thyroid enlargement), whereas functional thyroid diseases include thyroiditis, hyperthyroidism and hypothyroidism (Ron and Brenner, 2010). However, in clinical practice, alterations in thyroid hormone levels are frequently overlooked or misdiagnosed (Garmendia Madariaga et al., 2014). Women face a greater risk of such disorders 
than men, and the risk increases with age in both men and women (Kim and Park, 2014). Further, there are known environmental and dietary factors associated with thyroid disease risk, including cigarette smoking (Brent, 2010), fluoride and lithium exposure (Bocchetta et al., 2007; Singh et al., 2014), soy consumption and iodine deficiency (Brent, 2010). China has been identified as a region with high iodine intake (Zimmermann and Andersson, 2012) and a meta-analysis confirmed that excessive iodine intake increases the risk of thyroid diseases such as goitre and thyroid nodules (Stendel et al., 2020) in Chinese populations (Zhao et al., 2014).

One of the most toxic exposures to the human thyroid is radiation exposure (Ron and Brenner, 2010). The evidence for radiation exposure and thyroid cancer comes primarily from studies of atomic bomb survivors, accidental releases from nuclear power plants into the environment, and children with radiation treatment due to tinea capitis or enlargement of the thymus gland (AschebrookKilfoy et al., 2014). However, much less is known about the possible effects of occupational radiation exposure on thyroid hormone levels and the risk of non-malignant thyroid diseases such as hypothyroidism (Ron and Brenner, 2010), the most common endocrine disorder that is characterized by increased thyroid-stimulating hormone (TSH) levels (Vanderpump, 2011).

Occupational vibration exposure is common in many countries (Lin et al., 2005; EU-OSHA, 2008). In Europe, the proportion of workers exposed to vibration varies from $14 \%$ to $34 \%$ by country, with an average of one in three workers exposed to vibrations from some source (EU-OSHA, 2008). In China, it is estimated that two million workers use vibration tools (Lin et al., 2005), and vibration syndrome has been reported in 16 provinces in China (Xiao and Zheng, 1996). Vibration exposure is classified either as whole-body vibration (WBV) or hand-to-arm vibration (HAV) (Kraja et al., 2019). HAV syndrome (HAVS) is a condition associated with the use of hand-held vibrating tools, which has vascular, neurologic, and musculoskeletal features (Heaver et al., 2011). The most well-established manifestation of HAVS is known as vibration white finger (VWF), a type of secondary Raynaud phenomenon (Hack et al., 1986). The long-term effects of WBV and HAV exposure have been well characterized and include disorders of peripheral circulatory and nervous systems as well as injuries of the muscular-skeletal system (Lin et al., 2005; Johanning, 2015). Farmers are exposed to WBV through the use of all-terrain vehicles in agriculture (Milosavljevic et al., 2010), and are also exposed to chemicals such as pesticides (Wang et al., 2017a) and organic solvents (Bunn et al., 2009) that may have thyroid toxic effects (Saad-Hussein et al., 2011; Hassani et al., 2014). Indeed, female workers exposed to organic solvents (formaldehyde, phenol, $n$-hexane and chloroform) within the pharmaceutical industry have been reported as having higher levels of TSH than non-exposed individuals (Hassani et al., 2014). However, to the best of our knowledge, no previous study has examined the relationship between occupational exposure to vibration and thyroid disease or TSH levels in young adults.

In this study, we examined TSH concentrations in relation to occupational exposure to radiation, vibration, and organic solvents in young adults from Project Environmental and LifEstyle FActors iN metabolic health throughout life-course Trajectories (ELEFANT) in Tianjin, China. We used structured questionnaires to capture occupational exposures, and logistic and linear regression to estimate the association between vibration and TSH levels. We report that only vibration exposure was associated with an increase in TSH levels. Further, we report that vibration exposure was associated with higher prevalence of thyroid disease in young adults, as were exposures to radiation and organic solvents. Our results showed, for the first time, that vibration exposure may increase TSH levels and the risk of thyroid disorders. Further studies are required to elucidate the mechanisms underlying these associations and relative risk by type of thyroid disease.

\section{MATERIALS AND METHODS}

\subsection{Study Population}

We utilized data sets from ELEFANT, a prospective study designed to examine the impact of environmental and lifestyle factors at different points in the lifecycle through three age-based cohorts (Baby, Young and Elderly) upon the risk of non-communicable diseases (Cayir et al., 2018; Guo et al., 2018; Xu et al., 2018; Barrow et al., 2019). A total of 124,286 participants from the Young ELEFANT cohort were used in this study, who were recruited between 2014 and 2015 through clinic visitations in Tianjin, China. Eligible participants were young adults aged between 
18 and 40 years old, who were able to speak and read Chinese and attended clinics in Tianjin for regular check-ups. Participants were asked to complete an interviewer-administered questionnaire. Information collected through the questionnaire included socio-demographic characteristics, family history of disease, dietary habits, passive and active smoking, and occupational exposure to chemicals, radiation and vibration. All procedures and study protocols were approved by the ethics committee of Tianjin Medical University. All the participants provided written informed consent.

\subsection{Assessment of Thyroid-stimulating Hormone Levels and Thyroid Diseases}

Blood was collected from all the participants of Young ELEFANT. Serum were separated from the clot in 2 hours within blood collection. TSH levels were measured in serum by automated immunometric assay (cobas e 411; Roche). The immunoassay used chemiluminescent detection. The assay used in this study was labelled "third generation" by the manufacturer. We used corresponding commercial control materials with low and high TSH concentrations as quality control for cobas e 411. Doppler ultrasonography (GE Vingmed Ultrasound, Horten, Norway) was performed to examine thyroid structure. Medical records were reviewed to collect clinical information about thyroid disease, including hypothyroidism, hyperthyroidism, goitre, thyroid nodules and thyroid cancer. Thyroid diseases were diagnosed by clinicians at the participating hospital sites. Thyroid isthmus palpation was performed to identify thickness or the presence of a lump within the anterior tracheal soft tissue upward from the sternal notch. Thyroid lateral and posterior palpation were performed upon both lobes. Goitre was categorised into one of three categories according to the Chinese Diagnostic Criterion for Endemic Goiter (WS 276-2007).

\subsection{Statistical Analyses}

Exploratory data analysis (EDA) was performed to assess data quality and consistency, and the distribution of the variables of interest. EDA is an approach analysing data sets with visual methods to explore the data and formulate hypotheses beyond the formal modelling or hypothesis-testing task. A total of 124,286 young adults were eligible, of whom 124,170 remained for analysis after data editing after data quality controls. We tested normality of dependent variables by computing Shapiro-Wilk and skewness/kurtosis test. Pearson's correlation coefficient ( $\mathrm{rP}$ ) was performed to assess correlations between TSH levels and age or body mass index (BMI). We performed linear regression models to assess the association between occupational exposures (radiation, vibration or organic solvent contact) and TSH concentrations. Logistic regression models were employed to examine the relationship between occupational exposures and thyroid disease risk. Both logistic and linear regression models were adjusted for potential confounders. We selected confounders based on biological plausibility, their influence on the model fit (adjusted $r^{2}$ ) or their effect on the association between occupational exposures and outcomes (thyroid disease-TSH). The biological plausibility linking occupational exposures and thyroid disease were the covariates playing important roles in pathogenesis which have been learnt well in previous studies. Directed acyclic graphs (DAGs) were also constructed. All statistical models were adjusted for the same set of confounders: age (continuous, years), education (five categories), BMI (continuous, $\mathrm{kg} \mathrm{m}^{-2}$ ), permanent residence (urban rural $^{-1}$ ), occupation (nine categories), active and passive smoking, and co-exposures. All the analyses were performed using RStudio software 3.3.1 (R Foundation for Statistical Computing, Vienna, Austria, http://www.r-project.org). All reported p-values are two-sided and deemed significant at $\alpha=0.05$.

\section{RESULTS}

\subsection{Characteristics of the Study Population}

The characteristics of the Young ELEFANT study population, stratified by gender, is shown in Table 1. The majority were of the Han ethnic group (97.9\%), lived in rural areas (63.7\%), and worked as farmers (52.6\%). Regarding education, $39.5 \%$ and $36.7 \%$ reported college/university degrees and junior high school as the highest level of education, respectively. Only a small number reported illiteracy or primary school as the highest level of education (1.1\%). Women were more likely to have received college-level education and to have rural residence compared to men. The 
Table 1. Characteristics of the study population within Young ELEFANT stratified by gender.

\begin{tabular}{|c|c|c|c|c|c|}
\hline Variable & & All $(n=124,170)$ & Men $(n=62,089)$ & Women $(n=62,081)$ & $\mathrm{p}$ \\
\hline Age, years & & $29(27,33)$ & $30(27,33)$ & $29(26,32)$ & $<2.2 e^{-16}$ \\
\hline Gender & & & $62,089(50.0)$ & 62,081 (49.9) & \\
\hline BMI, kg m² & & $23.44(21.0,26.0)$ & $24.6(22.5,26.9)$ & $22.0(20.0,24.2)$ & $<2.2 e^{-16}$ \\
\hline \multirow[t]{4}{*}{ Ethnic group } & Han & 121,532 (97.9) & 60757 (97.9) & 60,775 (97.9) & 0.20 \\
\hline & Hui & $1514(1.2)$ & $783(1.3)$ & $731(1.2)$ & \\
\hline & Man & $690(0.6)$ & $341(0.6)$ & $349(0.6)$ & \\
\hline & Other & $389(0.3)$ & $178(0.3)$ & $211(0.3)$ & \\
\hline \multirow[t]{2}{*}{ Residence } & Urban & $45,025(36.3)$ & $23,231(37.4)$ & $21,794(35.1)$ & $<2.2 e^{-16}$ \\
\hline & Rural & $79,145(63.7)$ & $38,858(62.6)$ & 40,287 (64.9) & \\
\hline \multirow[t]{5}{*}{ Education } & 1 & 1375 (1.1) & $647(1.0)$ & $728(1.2)$ & $2.53 e^{-16}$ \\
\hline & 2 & $45,551(36.7)$ & $22,936(37.0)$ & $22,615(36.5)$ & \\
\hline & 3 & 24,630 (19.8) & $12,825(20.7)$ & 11,805 (19.0) & \\
\hline & 4 & 49,032 (39.5) & $23,917(38.6)$ & $25,115(40.5)$ & \\
\hline & 5 & $3493(2.8)$ & $1722(2.8)$ & $1771(2.9)$ & \\
\hline \multirow[t]{9}{*}{ Occupation } & 1 & $65,313(52.6)$ & $32,441(52.3)$ & $32,872(53.0)$ & $<2.2 e^{-16}$ \\
\hline & 2 & $16,607(13.4)$ & 10,135 (16.3) & $6472(10.4)$ & \\
\hline & 3 & $25,330(20.4)$ & 11,968 (19.3) & $13,362(21.5)$ & \\
\hline & 4 & $5396(4.4)$ & $3193(5.1)$ & $2203(3.6)$ & \\
\hline & 5 & $232(0.2)$ & $61(0.1)$ & $171(0.3)$ & \\
\hline & 6 & $2804(2.3)$ & $329(0.5)$ & $2475(4.0)$ & \\
\hline & 7 & $5003(4.0)$ & $2266(3.7)$ & $2737(4.4)$ & \\
\hline & 8 & $1574(1.3)$ & $638(1.0)$ & $936(1.5)$ & \\
\hline & 9 & $1911(1.5)$ & $1058(1.7)$ & $853(1.4)$ & \\
\hline \multirow[t]{2}{*}{ Active smoking } & No & $104,817(84.4)$ & $43,567(70.2)$ & $61,250(98.7)$ & $<2.2 e^{-16}$ \\
\hline & Yes & $19,353(15.6)$ & $18,522(29.8)$ & $831(1.3)$ & \\
\hline \multirow[t]{2}{*}{ Passive smoking } & No & $86,924(70.0)$ & $40,469(65.2)$ & 46,455 (74.8) & $<2.2 e^{-16}$ \\
\hline & Yes & 37,071 (29.9) & $21,536(34.7)$ & $15,535(25.0)$ & \\
\hline
\end{tabular}

Median and IQR for continuous variables, and $\mathrm{n}$ and percentage for categorical variables. Education categories:

(1) illiteracy/primary school education; (2) junior high school education; (3) high school/specialist school/technical school education; (4) college/university degrees; (5) graduate degree and above. Occupation categories: (1) farmer; (2) manual worker; (3) teacher/civil servant/staff/cadre/accountant/designer; (4) self-employed/businessman/freelance;

(5) doctors/nurses/pharmaceutical industry personnel (Stendel et al., 2020); housework; (7) services; (8) unemployed/student;

(9) other.

percentages of both active and passive smokers were higher in men compared to women. Overall, the missing values in Young ELEFANT for covariates and exposures were less than $1 \%$, and missing data was found only for age $(n=18,0.014 \%)$, ethnic group $(n=45,0.036 \%)$, education $(n=89$, $0.072 \%)$, and passive smoking $(n=175,0.14 \%)$. No values were missing for the remaining covariates and exposures.

Thyroid disease cases were marginally older than healthy cases (mean ages: 31 vs. 29 years old, respectively). The prevalence of thyroid disease was higher in women compared to men ( $0.73 \%$ vs. $0.12 \%$; Table 1 ) and decreased with increasing BMI (data not shown). Disease prevalence was higher in young adults living in urban regions compared to rural residence (Table S1) and showed higher TSH levels than healthy cases (2.05 vs. $1.95 \mathrm{mIU} \mathrm{L}^{-1}$; Fig. S1).

\subsection{Occupational Exposures among the Study Participants}

We examined participants with exposure to electron radiation, organic solvents and vibration through their occupations. In our study participants, women were more frequently exposed to electron radiation than men $(0.9 \%$ vs. $0.8 \%, p=0.009)$, while men were more frequently exposed to organic solvents $(1.3 \%$ vs. $1.1 \%, p=0.0009)$ and vibration $\left(0.5 \%\right.$ vs. $\left.0.1 \%, p<2.2 e^{-16}\right)$ (Table 2$)$. Overall, the frequency of exposure to organic solvents $(1.2 \%)$ was higher than electron radiation (0.9\%) and vibration (0.3\%) (Table 2 ). 
Table 2. Occupational exposures among Young ELEFANT participants.

\begin{tabular}{llllll}
\hline Exposure & & All $(\mathrm{n}=124,170)$ & Men $(\mathrm{n}=62,089)$ & Women $(\mathrm{n}=62,081)$ & $\mathrm{p}$ \\
\hline Electron radiation & No & $123,093(99.1)$ & $61,593(99.2)$ & $61,499(99.1)$ & $\mathbf{0 . 0 0 9}$ \\
& Yes & $1078(0.9)$ & $496(0.8)$ & $582(0.9)$ & $\mathbf{0 . 0 0 0 9}$ \\
Organic solvent & No & $122,711(98.8)$ & $61,296(98.7)$ & $61,415(98.9)$ & $<66(1.1)$ \\
\multirow{4}{*}{ Vibration } & Yes & $1459(1.2)$ & $793(1.3)$ & $61,992(99.9)$ & $<2.2 e^{-16}$ \\
& No & $123,799(99.7)$ & $61,807(99.5)$ & $89(0.1)$ & $282(0.5)$ \\
\hline
\end{tabular}

Median and IQRs are displayed for continuous variables, and $\mathrm{n}$ and percentage for categorical variables.

Table 3. Thyroid-stimulating hormone (TSH; $\mathrm{mIU} \mathrm{L}^{-1}$ ) levels by exposure type in female Young ELEFANT participants.

\begin{tabular}{lllll}
\hline Exposures & & $\mathrm{n}$ & $\beta(95 \% \mathrm{Cl})$ & $\mathrm{p}^{a}$ \\
\hline Electron radiation & No & 61,499 & Reference & \\
& Yes & 582 & $0.1(-0.6-0.7)$ & 0.869 \\
Organic solvent & No & 61,415 & Reference & \\
& Yes & 666 & $0.3(-0.3-0.9)$ & 0.368 \\
Vibration & No & 61,992 & Reference & \\
& Yes & 89 & $\mathbf{2 . 8 ( 1 . 1 - 4 . 5 )}$ & $\mathbf{0 . 0 0 1 6 *}$ \\
\hline
\end{tabular}

${ }^{a}$ Multivariate linear regression models adjusted for age, education, BMI, permanent residence, occupation, active and passive smoking, and co-exposures.

\subsection{Thyroid-stimulating Hormone Levels and Occupational Exposures}

Data on TSH levels in blood were available for female participants within Young ELEFANT as part of wider work within the ELEFANT study. We leveraged this data to examine associations between occupational exposures and TSH levels. TSH levels were higher among participants living in urban regions compared to rural ones ( $2.0 \mathrm{vs.} 1.9 \mathrm{mIU} \mathrm{L}^{-1}$, respectively; Table S1). The highest TSH concentrations were found in the Hui ethnic group (median: $2.13 ; 95 \% \mathrm{Cl}$ : 1.48-3.05), whilst the lowest were found in the Man ethnic group (median: 1.87; 95\% Cl: 1.22-2.70) (Table S1). TSH concentration was positively associated with BMI ( $\mathrm{rP}=0.019 ; \mathrm{p}<0.0001)$ but was not correlated with age $(r P=0.006 ; p=0.14)$.

In logistic regression analyses, the associations between exposures (electron radiation, organic solvent, and vibration) and TSH level were assessed. Women with occupational vibration exposure showed higher TSH levels $\left(2.8 \mathrm{mIU} \mathrm{L}^{-1}, 95 \% \mathrm{Cl}: 1.1-2.5\right)$ than those without exposure (Table 3 and Fig. 1). However, TSH concentrations were not associated with exposure to electron radiation or organic solvent contact in female study participants (Table 3 ).

\subsection{Thyroid Disease Prevalence and Occupational Exposures}

We conducted logistic regression analyses to assess the associations between different types of exposure and thyroid disease (Table 4). To maximise statistical power, we included hypothyroidism, hyperthyroidism, goitre, thyroid nodules and thyroid cancer in this analysis. The analysis was adjusted for age, education, BMI, permanent residence, occupation type, active and passive smoking, and co-exposures. Increased prevalence of thyroid disease was significantly associated with vibration exposure (OR: $4.4 ; 95 \% \mathrm{Cl}: 2.0-8.8$ ), electron radiation exposure (OR: $1.7 ; 95 \% \mathrm{Cl}$ : 1.1-2.7), and organic solvent contact (OR: 2.0; 95\% Cl: 1.3-2.9). We did not observe higher prevalence of thyroid disease in relation to overheating contact (data not shown).

\section{DISCUSSION}

Our results have revealed that occupational exposure to radiation, organic solvents and vibration increase the risk of thyroid disease in young adults. Consistent with previous studies, our results revealed TSH tends to increase with age due to intrinsic change of thyroid metabolism and 


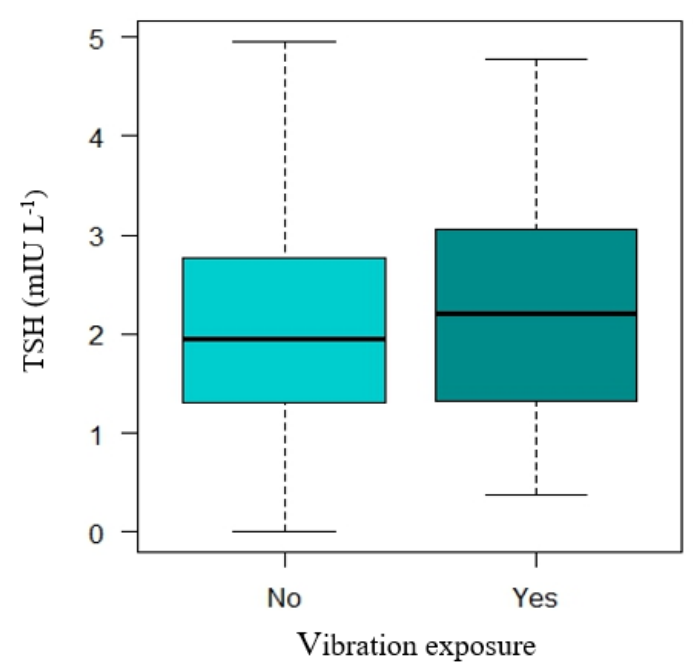

Fig. 1. Thyroid-stimulating hormone level by the exposure to vibration. TSH levels $\left(\mathrm{mIU} \mathrm{L}^{-1}\right)$ in female study participants with and without occupational vibration exposure. Medians are indicated by lines, while boxes represent the interquartile range and whiskers, the maximum and minimum values.

Table 4. Odds ratio (OR) of thyroid disease prevalence by occupational exposures in Young ELEFANT participants.

\begin{tabular}{lllll}
\hline Exposure & & $\mathrm{n}(\%)$ & OR $(95 \% \mathrm{Cl})$ & $\mathrm{p}^{a}$ \\
\hline Electron radiation & No & $507(96.0)$ & Reference & \\
& Yes & $21(4.0)$ & $\mathbf{1 . 7}(\mathbf{1 . 1 - 2 . 7 )}$ & $\mathbf{0 . 0 1 7}$ \\
Organic solvent & No & $500(94.7)$ & Reference & \\
& Yes & $28(5.3)$ & $\mathbf{2 . 0 ( 1 . 3 - 2 . 9 )}$ & $\mathbf{0 . 0 0 1}$ \\
Vibration & No & $518(98.1)$ & Reference & \\
& Yes & $10(1.9)$ & $\mathbf{4 . 4}(\mathbf{2 . 0 - 8 . 8 )}$ & $<\mathbf{0 . 0 0 1}$ \\
\hline
\end{tabular}

${ }^{a}$ The logistic regression models were adjusted for age, education, BMI, permanent residence, occupation, active and passive smoking, and co-exposures.

hypothalamic-pituitary-thyroid axis resetting gradually (Duntas, 2018). Among our population, we found significant differences on thyroid disease/TSH levels by gender. It has been reported that females have higher prevalence of all forms of thyroid diseases than male, although the underlying mechanism has not yet been clarified. (Gessl et al., 2012). Multiple studies showed that BMI is associated with serum TSH increased especially in obesity (Dai et al., 2020; Habib et al., 2020). TSH, as an important indicator of thyroid function, correlated with alterations in body weight. Leptin and lipid profile have been suggested to play important role linking TSH and BMI but still need to be further clarified (Betry et al., 2015; Wang et al., 2017b).

Furthermore, vibration exposure was associated with an increase in TSH concentrations in women. To the best of our knowledge, no previous studies have evaluated the risk of thyroid disease in relation to occupational vibration exposure, and there have been scarce studies examining the effect of vibration on thyroid hormone levels. Harada and colleagues reported that plasma thyroid hormone levels (triiodothyronine [T3] and thyroxine [T4]) are increased in people with vibration syndrome in response to cold exposure (Harada et al., 1990), while experimental evidence suggests that mechanical stimulation can influence TSH sensitivity in thyroid epithelial cells (Wagner et al., 2016). In our study, we observed an increase in TSH concentrations in women exposed to vibration compared to non-exposed participants. The mean TSH level in female participants within ELEFANT $\left(2.0 \mathrm{mIU} \mathrm{L^{-1 }}\right)$ were above the median of $1.3 \mathrm{mIU} \mathrm{L}^{-1}$ reported for apparently healthy Chinese adults (Quinn et al., 2009), which may be on account of the high prevalence of rural residence and occupations involving manual and farm work, and may suggest these individuals are at particular risk of thyroid disorders. Increases in TSH levels are often 
suggestive of hypothyroidism (Vanderpump, 2011), but the concentrations of T3 and T4 are also required to establish such a diagnosis. Hypothyroidism is characterized by a reduced sensitivity of thyroid epithelial cells to TSH; therefore, pituitary gland increased the release TSH to increase the release of T3 and T4 (Boron, 2012), which are critical for regulating cell metabolism, growth, and development (Boron, 2012). The mechanism underlying the association between vibration exposure and TSH levels may involve oxidative stress. Experimental studies have shown that vibration exposure results in an increase in the generation of reactive oxygen species (ROS) in the artery and nerves (Waugh et al., 2016). ROS production may increase TSH levels through mechanisms that involve reduced TSH sensitivity and reduced conversion of T4 to T3 by deiodinases (Mancini et al., 2016). Further studies are needed to evaluate levels of T3 and T4 in relation to vibration exposure and oxidative stress, and the subsequent functional consequences.

The evidence regarding thyroid disease risk in relation to radiation exposure within the workplace has primarily focused on medical staff, and the results have been inconsistent (Antonelli et al., 1995; Kim and Woo, 2016; Chen et al., 2017). For example, Chen et al. (2017) reported that physicians possibly exposed to medical radiation had higher risk of thyroid cancer. Similarly, medical workers exposed to chi radiation have been reported to be at higher risk of thyroid nodules than non-exposed individuals (Antonelli et al., 1995). However, contrary to these studies, Kim and Woo (2016) reported that the prevalence of thyroid nodules and thyroid cancer are not significantly higher among nurses compared with health check-up female participants. The inconsistency between different studies may originate from the lack of accurate individual estimates of radiation dose. In our study setting, medical staff (doctors, nurses, and pharmaceutical industry personnel) reported the highest percentage of radiation exposure (10.5\%), but the risk of thyroid disease was not higher among medical staff compared to other occupations (data not shown).

Our results showed higher risk of thyroid disease associated with organic solvent exposure at work. Epidemiologic studies have shown an increase in thyroid hormone levels in workers exposed to organic solvents (Uzma et al., 2008; Saad-Hussein et al., 2011; Hassani et al., 2014). For instance, Uzma et al. (2008) reported that petrol-filling workers exposed to organic solvents had higher levels of thyroxine (T4), free thyroxine (T4F) and lower levels of TSH and triiodothyronine (T3) compared to the non-exposed group. Workers exposed to paint production chemicals (primarily organic solvents) had higher levels of T3 and T4 and lower levels of antioxidants than the control group (Saad-Hussein et al., 2011). Further, TSH levels have been reported to be higher in women exposed to organic solvents in the pharmaceutical industry than the non-exposed group (4.2 vs. $2.4 \mathrm{ulU} \mathrm{mL}^{-1}$ ) (Hassani et al., 2014). In our study population, exposure to organic solvents was not significantly associated with TSH levels. However, we cannot discard the possibility that other thyroid hormones such as T3 and T4 might be modified by such exposures. Further work is required to elucidate the mechanisms and the type of thyroid disease that are associated with electron radiation and organic solvent contact.

The results of our study should be interpreted in light of the strengths and limitations of the work. A key strength of our study is the large sample size that is required to study risk factors associated with low-prevalence diseases such as thyroid disorders. Indeed, their low prevalence represented a significant challenge to this study, and subsequently we have been unable to analyse our data by more refined category of thyroid disease. A further strength of our work is that we have been able to leverage TSH measurements in a large number of subjects to gain some mechanistic insight into associations with thyroid pathology. However, the absence of TSH measurement in men represents a limitation, as does the absence of data on T3 and T4 concentrations. Half of our study population reported to be farmers and therefore may be exposed to other chemicals with thyroid toxic effects such as pesticides (Freire et al., 2013) that might influence our results. Despite these challenges, our work has for the first time revealed a link between vibration exposure and thyroid disease. If confirmed in other cohorts, thyroid examinations should be conducted in workers exposed to vibration within the workplace for early diagnosis and management of such disorders in an at-risk population.

\section{CONCLUSIONS}

This study analysed data sets from a large-scale project in Tianjin to evaluate vibration exposure 
as a risk factor for thyroid disease in young adults. We observed higher TSH levels in the exposed female participants than the non-exposed participants, and the mean TSH concentration for all of the female participants in our study $\left(2.0 \mathrm{mIU} \mathrm{L}^{-1}\right)$ exceeded the median reported for apparently healthy Chinese adults $\left(1.3 \mathrm{mIU} \mathrm{L}^{-1}\right)$. Additionally, the TSH concentrations in the female participants exhibited a correlation solely with the vibration exposure ( $\beta$ : $2.80 ; 95 \% \mathrm{Cl}$ : $1.07-4.54)$. Occupational exposure to vibration (OR: $4.4 ; 95 \% \mathrm{Cl}: 2.0-8.75)$, radiation (OR: $1.73 ; 95 \% \mathrm{Cl}: 1.1-2.7$ ) or organic solvents (OR: $2.0 ; 95 \% \mathrm{Cl}: 1.3-2.9$ ) also displayed an association with an increased risk of thyroid disease. The substantial evidence we found on the adverse effects of such exposure suggests the necessity of addressing these occupational hazards, especially as risk factors for thyroid dysfunction or disease. Further work is required to elucidate the mechanisms underpinning our observations.

\section{ACKNOWLEDGMENTS}

We gratefully acknowledge the staff of the study hospitals, the participants and their families. This research was funded in part by the National Natural Science Foundation of China, Grant Number 81971416 for L.G. and Tianjin Natural Science Foundation, Grant Number 18JCQNJC11700 for L.G. and China Postdoctoral Science Foundation, and Grant Number $2020 \mathrm{M670667}$ and the Secretary of Science, Technology and Innovation (SECITI-DF), Mexico, BP15-001 for C.O.Y. The APC was funded by the National Natural Science Foundation of China.

\section{CONFLICTS OF INTEREST}

The authors declare no conflict of interest.

\section{SUPPLEMENTARY MATERIAL}

Supplementary material for this article can be found in the online version at https://doi. org/10.4209/aaqr.210085

\section{REFERENCES}

Antonelli, A., Silvano, G., Bianchi, F., Gambuzza, C., Tana, L., Salvioni, G., Baldi, V., Gasperini, L., Baschieri, L. (1995). Risk of thyroid nodules in subjects occupationally exposed to radiation: A cross sectional study. Occup. Environ. Med. 52, 500-504. https://doi.org/10.1136/oem.52.8.500

Aschebrook-Kilfoy, B., Ward, M.H., Della Valle, C.T., Friesen, M.C. (2014). Occupation and thyroid cancer. Occup. Environ. Med. 71, 366-380. https://doi.org/10.1136/oemed-2013-101929

Barrow, T.M., Peng, C., Wilson, A., Wang, H., Liu, H., Shen, L., Tang, N.J., Sae-Lee, C., Li, P.H., Guo, L., Byun, H.M. (2019). Psychosocial stress is associated with benign breast disease in young Chinese women: RESULTS from Project ELEFANT. Breast Cancer Res. Treat. 173, 217-224. https://doi.org/10.1007/s10549-018-4979-4

Betry, C., Challan-Belval, M.A., Bernard, A., Charrie, A., Drai, J., Laville, M., Thivolet, C., Disse, E. (2015). Increased TSH in obesity: Evidence for a BMI-independent association with leptin. Diabetes Metab. 41, 248-251. https://doi.org/10.1016/j.diabet.2014.11.009

Bocchetta, A., Cocco, F., Velluzzi, F., Del Zompo, M., Mariotti, S., Loviselli, A. (2007). Fifteen-year follow-up of thyroid function in lithium patients. J. Endocrinol. Invest. 30, 363-366. https://doi.org/10.1007/BF03346311

Boron, W.F. (2009). Medical physiology: A cellular and molecular approach, 2nd ed. Saunders/Elsevier, Philadelphia.

Brent, G.A. (2010). Environmental exposures and autoimmune thyroid disease. Thyroid 20, 755761. https://doi.org/10.1089/thy.2010.1636

Bunn, T.L., Liu, Y., Lee, K., Robertson, M., Yu, L. (2009). Farmer exposure to organic solvents during the maintenance and repair of farm machinery: a pilot study. Am. J. Ind. Med. 52, 973981. https://doi.org/10.1002/ajim.20773 
Cayir, A., Barrow, T.M., Wang, H., Liu, H., Li, C., Ding, N., Li, Y., Kang, C.M., Guo, L., Li, P.H., Byun, H.M. (2018). Occupational noise exposure is associated with hypertension in China: Results from project ELEFANT. PLoS One 13, e0209041. https://doi.org/10.1371/journal.pone.0209041

Chen, T.Y., Hsu, C.C., Feng, I.J., Wang, J.J., Su, S.B., Guo, H.R., Huang, C.C., Lin, H.J. (2017). Higher risk for thyroid diseases in physicians than in the general population: A Taiwan nationwide population-based secondary analysis study. QJM 110, 163-168. https://doi.org/10.1093/qjme d/hcw140

Dai, H.Y., Zhang, L., Han, X., Zhao, H.Y., Guo, J.L., Li, Z.W., Yang, A.J. (2020). Body mass index (BMI) is associated with serum thyroid-stimulating hormone (TSH) level in infertile women: A crosssectional study. Endocr. J. 67, 923-928. https://doi.org/10.1507/endocrj.EJ19-0441

Duntas, L.H. (2018). Thyroid function in aging: A discerning approach. Rejuvenation Res. 21, 2228. https://doi.org/10.1089/rej.2017.1991

EU-OSHA (2008). European Agency for Safety and Health at Work. An expert review on Work Place Exposure to Vibration in Europe.

Freire, C., Koifman, R.J., Sarcinelli, P.N., Simoes Rosa, A.C., Clapauch, R., Koifman, S. (2013). Long-term exposure to organochlorine pesticides and thyroid status in adults in a heavily contaminated area in Brazil. Environ. Res. 127, 7-15. https://doi.org/10.1016/j.envres.2013.09.001

Garmendia Madariaga, A., Santos Palacios, S., Guillen-Grima, F., Galofre, J.C. (2014). The incidence and prevalence of thyroid dysfunction in Europe: A meta-analysis. J. Clin. Endocrinol. Metab. 99, 923-931. https://doi.org/10.1210/jc.2013-2409

Gessl, A., Lemmens-Gruber, R., Kautzky-Willer, A. (2012). Thyroid Disorders, in: Regitz-Zagrosek, V. (Eds.), Sex and Gender Differences in Pharmacology. Vol. 214, Springer Berlin Heidelberg, Berlin, pp. 361-386. https://doi.org/10.1007/978-3-642-30726-3_17

Guo, L., Peng, C., Xu, H., Wilson, A., Li, P.H., Wang, H., Liu, H., Shen, L., Chen, X., Qi, X., Tang, N.J., Barrow, T.M., Byun, H.M. (2018). Age at menarche and prevention of hypertension through lifestyle in young Chinese adult women: Result from project ELEFANT. BMC Womens Health. 18, 182. https://doi.org/10.1186/s12905-018-0677-y

Habib, A., Molayemat, M., Habib, A. (2020). Elevated serum TSH concentrations are associated with higher BMI Z-scores in southern Iranian children and adolescents. Thyroid Res. 13, 9. https://doi.org/10.1186/s13044-020-00084-9

Hack, M., Boillat, M.A., Schweizer, C., Lob, M. (1986). Assessment of vibration induced white finger: Reliability and validity of two tests. Br. J. Ind. Med. 43, 284-287. https://doi.org/10.113 6/oem.43.4.284

Harada, N., Nakamoto, M., Kohno, H., Kondo, H., Tanaka, M. (1990). Hormonal responses to cold exposure in subjects with vibration syndrome. Kurume Med. J. 37, S45-S52. https://doi.org/1 0.2739/kurumemedj.37.SUPPLEMENT_S45

Hassani, S., Namvar, M., Ghoreishvandi, M., Attarchi, M., Golabadi, M., Seyedmehdi, S.M., Khodarahmian, M. (2014). Menstrual disturbances and hormonal changes in women workers exposed to a mixture of organic solvents in a pharmaceutical company. Med. J. Islam. Repub. Iran 28, 156.

Heaver, C., Goonetilleke, K.S., Ferguson, H., Shiralkar, S. (2011). Hand-arm vibration syndrome: A common occupational hazard in industrialized countries. J. Hand Surg. 36, 354-363. https://doi.org/10.1177/1753193410396636

Johanning, E. (2015). Whole-body vibration-related health disorders in occupational medicine-An international comparison. Ergonomics 58, 1239-1252. https://doi.org/10.1080/00140139. 2015.1005170

Kim, K.H., Woo, S.H. (2016). An occupational study in nurses: Prevalence of thyroid nodules and cancer in comparison to health check-up female. Clin. Exp. Otorhinolaryngol. 9, 252-256. https://doi.org/10.21053/ceo.2015.01109

Kim, Y.A., Park, Y.J. (2014). Prevalence and risk factors of subclinical thyroid disease. Endocrinol. Metab 29, 20-29. https://doi.org/10.3803/EnM.2014.29.1.20

Kraja, A.T., Liu, C., Fetterman, J.L., Graff, M., Have, C.T., Gu, C., Yanek, L.R., Feitosa, M.F., Arking, D.E., Chasman, D.I., Young, K., Ligthart, S., Hill, W.D., Weiss, S., Luan, J., Giulianini, F., Li-Gao, R., Hartwig, F.P., Lin, S.J., Wang, L., Richardson, T.G., et al. (2019). Associations of mitochondrial and nuclear mitochondrial variants and genes with seven metabolic traits. Am. J. Hum. Genet. 104, 112-138. https://doi.org/10.1016/j.ajhg.2018.12.001 
Lin, W., Chunzhi, Z., Qiang, Z., Kai, Z., Xiaoli, Z. (2005). The study on hand-arm vibration syndrome in China. Ind. Health 43, 480-483. https://doi.org/10.2486/indhealth.43.480

Mancini, A., Di Segni, C., Raimondo, S., Olivieri, G., Silvestrini, A., Meucci, E., Curro, D. (2016). Thyroid Hormones, Oxidative Stress, and Inflammation. Mediators Inflammation 2016, 6757154. https://doi.org/10.1155/2016/6757154

Milosavljevic, S., Bergman, F., Rehn, B., Carman, A.B. (2010). All-terrain vehicle use in agriculture: Exposure to whole body vibration and mechanical shock. Appl. Ergon. 41, 530-535. https://doi.org/10.1016/j.apergo.2009.11.002

Quinn, F.A., Tam, M.C., Wong, P.T., Poon, P.K., Leung, M.S. (2009). Thyroid autoimmunity and thyroid hormone reference intervals in apparently healthy Chinese adults. Clin. Chim. Acta 405, 156-159. https://doi.org/10.1016/j.cca.2009.04.021

Ron, E., Brenner, A. (2010). Non-malignant thyroid diseases after a wide range of radiation exposures. Radiat. Res. 174, 877-888. https://doi.org/10.1667/RR1953.1

Saad-Hussein, A., Hamdy, H., Aziz, H.M., Mahdy-Abdallah, H. (2011). Thyroid functions in paints production workers and the mechanism of oxidative-antioxidants status. Toxicol. Ind. Health 27, 257-263. https://doi.org/10.1177/0748233710386409

Singh, N., Verma, K.G., Verma, P., Sidhu, G.K., Sachdeva, S. (2014). A comparative study of fluoride ingestion levels, serum thyroid hormone \& TSH level derangements, dental fluorosis status among school children from endemic and non-endemic fluorosis areas. SPRINGERPLUS 3, 7. https://doi.org/10.1186/2193-1801-3-7

Stendel, C., Neuhofer, C., Floride, E., Shi, Y.Q., Ganetzky, R.D., Park, J., Freisinger, P., Kornblum, C., Kleinle, S., Schols, L., Distelmaier, F., Stettner, G.M., Buchner, B., Falk, M.J., Mayr, J.A., Synofzik, M., Abicht, A., Haack, T.B., Prokisch, H., Wortmann, S.B., et al. (2020). Delineating MT-ATP6associated disease: From isolated neuropathy to early onset neurodegeneration. Neurol. Genet. 6, e393. https://doi.org/10.1212/NXG.0000000000000393

Uzma, N., Salar, B.M., Kumar, B.S., Aziz, N., David, M.A., Reddy, V.D. (2008). Impact of organic solvents and environmental pollutants on the physiological function in petrol filling workers. Int. J. Env. Res. Public Health 5, 139-146. https://doi.org/10.3390/ijerph5030139

Vanderpump, M.P. (2011). The epidemiology of thyroid disease. Br. Med. Bull. 99, 39-51. https://doi.org/10.1093/bmb/ldr030

Wagner, A.P., Chinnathambi, S., Titze, I.R., Sander, E.A. (2016). Vibratory stimulation enhances thyroid epithelial cell function. Biochem. Biophys. Rep. 8, 376-381. https://doi.org/10.1016/j. bbrep.2016.10.008

Wang, W., Jin, J., He, R., Gong, H. (2017a). Gender differences in pesticide use knowledge, risk awareness and practices in Chinese farmers. Sci. Total Environ. 590-591, 22-28. https://doi.org/ 10.1016/j.scitotenv.2017.03.053

Wang, Y.Q., Yin, Q.L., Xu, M., Ni, Q.C., Wang, W.Q., Wang, Q.D. (2017b). BMI modulates the effect of thyroid hormone on lipid profile in euthyroid adults. Int. J. Endocrinol. 2017, 8591986. https://doi.org/10.1155/2017/8591986

Waugh, S., Kashon, M.L., Li, S., Miller, G.R., Johnson, C., Krajnak, K. (2016). Transcriptional pathways altered in response to vibration in a model of hand-arm vibration syndrome. J. Occup. Environ. Med. 58, 344-350. https://doi.org/10.1097/JOM.0000000000000705

Xiao, J.M., Zheng, F.Y. (1996). Hand-arm vibration hazard and prevention in China. Cent. Eur. J. Public Health 4, 40-42.

Xu, H., Li, P.H., Barrow, T.M., Colicino, E., Li, C., Song, R., Liu, H., Tang, N.J., Liu, S., Guo, L., Byun, H.M. (2018). Obesity as an effect modifier of the association between menstrual abnormalities and hypertension in young adult women: Results from Project ELEFANT. PLoS One 13, e0207929. https://doi.org/10.1371/journal.pone.0207929

Zhao, W., Han, C., Shi, X., Xiong, C., Sun, J., Shan, Z., Teng, W. (2014). Prevalence of goiter and thyroid nodules before and after implementation of the universal salt iodization program in mainland China from 1985 to 2014: a systematic review and meta-analysis. PLoS One 9, e109549. https://doi.org/10.1371/journal.pone.0109549

Zimmermann, M.B., Andersson, M. (2012). Update on iodine status worldwide. Curr. Opin. Endocrinol. Diabetes Obes. 19, 382-387. https://doi.org/10.1097/MED.0b013e328357271a 